\title{
Acceso y satisfacción con servicios curativos: análisis de casos en afiliados al régimen contributivo y afiliados al régimen subsidiado en un barrio estrato 2 - Cali, Colombia.
}

\section{Access and satisfaction related to curative services: Case analysis of the contributory regime and members of the subsidized regime in a strata 2 neighborhood. Cali, Colombia}

\section{Acesso e satisfação com serviços curativos: Análise de casos em filiados ao régime contributivo e filiados ao régime subsidiado em um bairro de estrato socioeconômico 2 . Cali, Colômbia}

Fecha de recepción: 15-05-15 Fecha de aceptación: 24-08-15 Disponible en línea: 01-11-15 doi: 10.11144/Javeriana.rgyps14-29.assc

Cómo citar este artículo:

Díaz Grajales C, Zapata Bermúdez Y, Aristizábal Grisales JC. Acceso y satisfacción con servicios curativos: Análisis de casos en afiliados al régimen contributivo y afiliados al régimen subsidiado en un barrio estrato 2. Cali, Colombia. Rev. Gerenc. Polít. Salud. 2015; 14(29): 155-178. http://dx.doi.org/10.11144/ Javeriana.rgyps14-29.assc

\author{
Constanza Díaz-Grajales** \\ Yolanda Zapata-Bermúdez*** \\ Juan Carlos Aristizábal-Grisales****
}

" Artículo de investigación. Se presenta una parte del estudio Diferencias en el acceso a servicios de salud entre población afiliada al régimen contributivo y subsidiado estrato 2 de Cali, desarrollada como trabajo de grado de la Maestría en Salud Pública, entre enero y diciembre del 2009, cofinanciado por la Vicerrectoría de Investigaciones de la Universidad del Valle a los grupos de investigación Cedetes y Cemiya. Un artículo sobre las diferencias en el acceso a los servicios preventivos fue publicado en el 2011 en el número 21 de esta revista.

*** Magíster en Salud Pública, profesora de la Escuela de Salud Pública e investigadora de Cedetes, Universidad del Valle. Correo electrónico: constanza.diaz@correounivalle.edu.co. Correspondencia: Escuela de Salud Pública, Universidad del Valle, Calle 4 B \# 36-00 Edificio Santiago Renjifo, Cali, Colombia.

**** Magíster en Salud Pública, directora adjunta de la Especialización en Administración en Salud y docente del Departamento de Gestión de Organizaciones de la Facultad de Ciencias Económicas y Administrativas. Investigadora y miembro del grupo de investigación Salud y Calidad de Vida de la Pontificia Universidad Javeriana de Cali. Correo electrónico: yzapata@javerianacali.edu.co.

***** Magíster en Epidemiología, investigador y miembro del Grupo de Investigación en Desarrollo Regional de la Facultad de Ciencias Económicas y Administrativas y docente del Departamento de Salud Pública y Epidemiología de la Facultad de Ciencias de la Salud de la Pontificia Universidad Javeriana Cali. Correo electrónico: jcaristizabal@javerianacali.edu.co. 


\section{Resumen}

Este estudio presenta un análisis de casos para identificar diferencias en el acceso a servicios curativos entre afiliados al régimen contributivo y afiliados al régimen subsidiado. A nivel poblacional, alrededor de una décima parte de la población siente la necesidad y demanda un servicio curativo, y se atiende al $97,8 \%$. Se trata de una investigación descriptiva de corte transversal, las variables fundamentales se operacionalizaron teniendo en cuenta la necesidad atendida. Se consideraron casos quienes presentaron un problema de salud en el último mes, consultaron un servicio formal y fueron atendidos. Los resultados muestran diferencias entre los regímenes en el acceso y en la satisfacción; por ejemplo, con relación a medicamentos, los reclamaron el $87,8 \%$ del régimen contributivo y el 55,9\% del régimen subsidiado, diferencias estadísticamente significantes. Se encontraron barreras administrativas y económicas y características de los servicios que afectan el acceso a la atención y la calidad en ambos regímenes, y se evidenció vulneración del derecho fundamental a la atención en salud.

Palabras clave: seguridad social; accesibilidad a los servicios de salud; necesidades y demandas de servicios de salud; atención médica; utilización; satisfacción del paciente

\section{Abstract}

This study presents a case analysis to identify differences in access to curative services among people affiliated to the contributive and subsidized system. At the population level, around a tenth of the population feels the need and demand a healing service, $97.8 \%$ being treated. Descriptive, cross-sectional investigation; fundamentals variables were operationalized taking into account the need serviced. Cases were considered, those presenting a health problem in the last month, consulted a formal service and were treated. The results show differences between the regimes of access and satisfaction; for example, in relation to the medicines they claimed $87.8 \%$ of contributive and subsidized $55.9 \%$, statistically significant differences. Administrative and financial barriers were found; and characteristics of the services that affect access to care and quality in both regimes, demonstrating infringement of the fundamental right to health care.

Keywords: social security; health services accessibility; health services needs and demand; medical care; utilization; patient satisfaction

\section{Resumo}

Este estudo apresenta análise de casos para identificar diferenças no acesso a serviços curativos entre filiados ao regime contributivo e filiados ao regime subsidiado. No nível populacional, perto de uma décima parte da população sente necessidade e demanda cualquer serviço curativo, e é atendido o $97,8 \%$. Trata-se de pesquisa descritiva de tipo transversal, as variáveis fundamentais foram operacionalizadas levando em conta a necessidade atendida. Consideraram-se casos que apresentaram problema de saúde no último mês, pediram um serviço formal e foram atendidos. Os resultados mostram diferenças entre os regimes no acesso e satisfação; por exemplo, no que diz respeito a medicamentos, foram pegados pelo $87,8 \%$ do regime contributivo e o $55,9 \%$ do regime subsidiado, diferenças estatisticamente significantes. Encontraram-se barreiras administrativas e económicas e características dos serviços que afetam o acesso ao atendimento e qualidade em ambos os regimes e evidenciou-se violação do direito fundamental de atendimento em saúde.

Palavras-chave: segurança social; acessibilidade a serviços de saúde; necessidades e demandas de serviços de saúde; atendimento médico; utilização; satisfação do paciente 
ACCESO Y SATISFACCIÓN CON SERVICIOS CURATIVOS: ANÁLISIS DE CASOS EN AFILIADOS AL RÉGIMEN CONTRIBUTIVO Y afiliados al rÉgimen subsidiado en un barRio estrato 2. Cali, Colombia

\section{Introducción}

Una de las competencias sustantivas e indelegables de la autoridad sanitaria en la rectoría del sistema de salud es garantizar el acceso universal y equitativo a la atención de la salud con criterios de calidad (1). En Colombia, el modelo de operación del Sistema General de la Seguridad Social en Salud (sGsss), si bien ha tenido logros, especialmente en coberturas de afiliación, han sido mayores los innumerables cuestionamientos, tanto a nivel nacional como a nivel internacional, relacionados con los impactos del Sistema y en particular por las restricciones al acceso de la población a la atención de su salud (2-7).

Estos cuestionamientos, expresados permanentemente por los diferentes actores institucionales y sociales, llevaron al Congreso de la República a proponer un ajuste, a través de la Ley 1122 del 2007 (8) que tenía como prioridad el mejoramiento en la prestación de los servicios a los usuarios. Sin embargo, de esta norma solo se reglamentaron pocos aspectos, y los problemas continuaron tanto en el flujo de los recursos financieros como en el equilibrio entre los actores y especialmente en la atención de la salud. En este último aspecto ha sido de tal grado la magnitud de la inconformidad, que en el país se acuñó el término despectivo "paseo de la muerte" (9). Situación que no ha escapado a la Corte Constitucional, que tras muchos años de revisar cientos de tutelas que los colombianos interpusieron para recibir los tratamientos, medicamentos y procedimientos de salud que requerían, decidió obligar al Gobierno a buscar soluciones de fondo. Por eso, tras dos años de estudios y trabajos, expidió una histórica sentencia (10) con la que se busca eliminar las barreras que impiden el acceso a la salud de los colombianos, sentando jurisprudencia al determinar de manera clara que "la salud es un derecho fundamental", y no un derecho de segunda generación, lo que significa que debe ser garantizado a todas las personas.

Lo anterior justifica el desarrollo y la difusión de investigaciones como esta, que tuvo por objeto identificar, en una población de un mismo estrato, las diferencias de acceso a los servicios de salud tanto preventivos como curativos, para el régimen contributivo (RC) y para el régimen subsidiado (Rs). A este efecto se seleccionó un barrio de estrato socioeconómico 2 , teniendo en cuenta que en el municipio de Cali la población ubicada en este nivel socioeconómico corresponde aproximadamente al 31,2\% (11), una importante proporción de población vulnerable por atender.

Este artículo presenta los resultados del estudio relativos al acceso, la utilización y la satisfacción con los servicios curativos, que cobran vigencia en el contexto actual donde el debate con respecto al sGsss no acaba, a pesar de la última reforma decretada por la Ley Estatutaria 1751 del 2015, por la cual se regula el derecho fundamental a la salud (12).

Para validar la vigencia de la información obtenida en el año 2010 mediante el trabajo de campo, información publicada parcialmente en el año 2011 en un primer artículo sobre el acceso a servicios preventivos (13), se revisaron publicaciones en las cuales se hubieran realizado trabajos de campo y se hubieran obtenido datos sobre la temática en el periodo 2011-2014, que permitieran comparar los hallazgos de esta investigación en el componente de servicios curativos. A este respecto se encontraron dos artículos con información recogida en el año 2011 sobre inequidades en el acceso al cuidado continuo y comparación de factores que influyen en el acceso en dos municipios de Brasil y dos de Colombia $(14,15)$, y un estudio de caso, realizado en Medellín, que identificó barreras de acceso y estrategias desde la expectativa 
de los actores (16), cuyos resultados validan los hallazgos de esta investigación.

Otras publicaciones encontradas estaban relacionadas con: uso de información de encuestas nacionales con datos previos al 2010 (17), producto de revisiones bibliográficas (18-20) y sobre poblaciones o servicios específicos como desplazados (21), población en cárcel (22) o niños portadores de VIH (23), salud bucal (24), control prenatal (25), diagnóstico y tratamiento de anormalidades citológicas entre mujeres (26) y dispensación de medicamentos (27).

\section{Marco de referencia}

El concepto de acceso ha sido ampliamente discutido, los términos acceso y accesibilidad se han usado como sinónimos y se han definido ambiguamente (28). Se reconocen muchas definiciones, algunas de las más citadas corresponden a autores como Donabedian (29), Aday y Anderson (30), Anderson $(31,32)$, Penchansky y Thomas (33) y Frenk (34); recientemente, Levesque, Harris y Russell (35). Lo anterior coincide con el artículo de Tovar y Arrivillaga sobre el estado del arte de la investigación en acceso a servicios de salud en Colombia (20). El concepto de acceso se ha equiparado con "disponibilidad", "accesibilidad" y "búsqueda de atención”. El consenso es que el acceso a un determinado servicio no solo conlleva la disponibilidad de recursos, sino que comprende además la relevancia, oportunidad y adecuación de estos con las reales necesidades de la población (36).

La definición más clásica corresponde a Donabedian, quien consideró la accesibilidad como el grado de ajuste entre las características de la población y las de los recursos, con miras a la búsqueda y obtención de la atención; esta se evalúa en el marco de las interacción entre los usuarios y los recursos del sistema de salud (29). La calidad depende, entre otros aspectos, de la mayor o menor accesibilidad que se tenga a la atención en salud, y una vez se ha logrado, a la mayor o menor calidad de la atención proporcionada (37).

Con relación a los modelos explicativos sobre acceso, accesibilidad y utilización de los servicios de salud, Arredondo y Meléndez los categorizaron en cinco grupos: modelos epidemiológicos y sociales, psicosociales, sociales, económicos y sociológicos-psicológicos (38).

Dado que esta investigación tomó como marco referente para el análisis el modelo social de acceso de Aday y Andersen (30), uno de los más usados en la evaluación de acceso a servicios de salud (38), combinándolo con la propuesta de accesibilidad de Frenk (34), la cual, cuando presenta los posibles dominios del concepto de accesibilidad recoge aspectos de los modelos antes mencionados, para este estudio se adoptaron estos conceptos.

Para Aday y Andersen el acceso se entiende como las características y condiciones de la población y del sistema prestador de servicios que posibilitan la atención en salud (acceso potencial) y los aspectos relacionados con el ingreso efectivo al sistema y con el resultado de la interacción entre población y sistema prestador, como son la utilización de los servicios de atención médica y la satisfacción de las necesidades del usuario (acceso realizado) (30). Los autores consideran el acceso más como un concepto político que como un concepto operativo y presentan un modelo teórico de este, según el cual, los elementos que describen las características del sistema y de la población sirven como indicadores de proceso y la utilización y la conformidad de los consumidores como indicadores de resultado (30). 
ACCESO Y SATISFACCIÓN CON SERVICIOS CURATIVOS: ANÁLISIS DE CASOS EN AFILIADOS AL RÉGIMEN CONTRIBUTIVO Y afiliados al rÉgimen subsidiado en un barRio estrato 2. Cali, Colombia

Para Frenk, el término acceso denota "la capacidad de un individuo o grupo de individuos para buscar y obtener atención" (34). Acceso se refiere a una característica de la población de usuarios potenciales o reales de los servicios, y, argumentando que este concepto tan restringido probablemente tenga poca aceptación, el autor plantea un término alternativo, el de "poder de utilización”, análogo al de "poder de compra", y añade el concepto de "resistencia" como complementario. La resistencia se define como el conjunto de obstáculos a la búsqueda y obtención de la atención, los cuales se originan en los servicios de salud, y tiene su contrapartida en el poder de utilización - "acceso" - como característica de la población. El autor es cuidadoso en aclarar que este concepto se refiere a obstáculos distintos de la falta de capacidad de producir servicios, ya que el nivel de capacidad está comprendido en el concepto de disponibilidad. También expone el concepto de "disponibilidad efectiva de los recursos" como su disponibilidad corregida según la resistencia que oponen a la búsqueda y obtención de servicios. Entre los obstáculos o factores disuasivos están el costo de los servicios, la ubicación de las fuentes de atención a la salud y ciertas características de las formas en que se organizan los recursos, entre estas las demoras para obtener citas o para recibir atención.

\section{Materiales y métodos}

Este artículo presenta los resultados de una de las unidades de análisis del estudio general de identificación de las diferencias en el acceso a los servicios de salud, entre población afiliada al RC y población afiliada al Rs de un barrio estrato 2 de Cali.

La unidad de análisis fue definida como: los individuos que tuvieron un problema de salud y consultaron un servicio formal de salud en el último mes, contado este hasta la fecha de aplicación de la encuesta, con el objetivo de explorar el acceso a servicios de salud específicos, barreras de acceso a los servicios de salud consultados y satisfacción con los servicios recibidos. Esta exploración se abordó a través de la metodología de análisis de casos. Las variables fundamentales se operacionalizaron teniendo en cuenta la necesidad atendida, conceptualizada como la prestación de los servicios a los usuarios afiliados al RC o al RS que demandaron la atención.

Esta investigación fue de tipo descriptivo y corte transversal, se llevó a cabo entre 2009 y 2011 en el barrio República de Israel (BRI), clasificado como estrato socioeconómico 2, de la comuna 16 (C16) de Santiago de Cali. La población de referencia estuvo conformada por 16944 personas, distribuidas en 4847 hogares, para un promedio de 3,5 personas por hogar, residentes en 1602 viviendas,

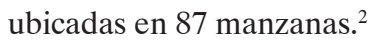

Para el cálculo del tamaño de muestra se asumió una precisión aceptable, representada en un error estándar relativo (Esrel) menor o igual al 3,0\%, una proporción de afiliación al régimen contributivo del $50 \%$, de acuerdo con información suministrada por la Secretaría de Salud Pública Municipal de Cali, un nivel de confiabilidad del $95 \%$ y un efecto de diseño (Deff) de 2, para un tamaño de muestra de 1965 personas, con un $20 \%$ adicional para compensar la tasa de no respuesta, obteniendo un tamaño final de 2357 personas. Se encuestaron en total 2165 personas, muestra suficiente para todos los requerimientos de estimación estadística en este estudio.

1 Para el año 2009, según Planeación Municipal, todos los lados de manzana estaban clasificados en estrato 2 .

2 Según el Censo 2005 del Departamento Administrativo Nacional de Estadística (DANE), descrito en Cali en Cifras 2007. 
Para el diseño del cuestionario fueron revisados instrumentos validados que actualmente son aplicados a nivel nacional por entes gubernamentales tales como el Departamento Nacional de Planeación y el Ministerio de Salud y Protección Social (MSPS). Este se aplicó en una muestra obtenida mediante muestreo probabilístico complejo (estratificado y polietápico) (39), previo muestreo piloto, con el fin de examinar el instrumento y los criterios de muestreo. Como consecuencia del tipo de muestreo utilizado y antes de desarrollar el procesamiento estadístico de la información, se calcularon los correspondientes pesos de muestreo y factores de expansión; ${ }^{3}$ adicionalmente, se calculó el factor de ajuste por post-estratificación (40), que restituye a los totales poblacionales por edad y sexo (41) de la población referencia DANE para la zona.

El análisis de la información se llevó a cabo en dos etapas, en primera instancia fue desarrollado el análisis univariado y posteriormente el análisis bivariado y estratificado, para establecer la relación de las variables entre sí, de acuerdo con los objetivos del estudio. Adicionalmente, se aplicaron metodologías de georreferenciación para realizar el mapeo de las instituciones prestadoras del servicio de salud que usan las personas inscritas al RC y las personas inscritas al Rs, con relación al barrio en donde se realizó la investigación.

Para identificar los casos se establecieron los siguientes criterios de inclusión: 1) pertenecer a un hogar con integrantes afiliados al rc o al rs de cualquier edad y sexo, 2) contar con un informante idóneo y 3 ) haber tenido un problema de salud y haber usado un

3 Factor que le proporciona a la muestra el peso proporcional a la población investigada. Dicho factor se obtiene mediante el cálculo que considera las unidades primarias de muestreo y las probabilidades de selección de cada una de ellas, que conforman la muestra. servicio formal de salud en el último mes y hasta la fecha de la encuesta. Como criterio de exclusión se estableció: no considerar a los hogares donde ninguno de sus integrantes cuenta con algún tipo de afiliación al sGsss.

Entre las personas del hogar que cumplieron los criterios se seleccionó un individuo al azar; estos correspondieron a un $66 \%$ de las personas de la muestra que cumplieron los criterios.

Los resultados en este artículo abordan de manera específica las variables relacionadas con el acceso a la consulta médica general, y también otro tipo de servicios como las pruebas diagnósticas, la consulta por especialistas, el suministro de medicamentos, las hospitalizaciones y los servicios de urgencias, de manera comparativa por régimen de afiliación. En particular, se indagó por la satisfacción del usuario en cada uno de los servicios de atención a los que accedió. En este sentido, es importante mencionar que se hicieron procesos de medición utilizando categorías suficientes, de tal manera que permitieran expresar dicho nivel de satisfacción. Posteriormente, estas categorías fueron reagrupadas para generar las categorías de "Satisfecho" o "Insatisfecho" y asî realizar, a través del uso de procedimientos de estadística inferencial, la valoración de la relación entre el nivel de satisfacción y el tipo de afiliación al sGsss. Esto se hizo para cada uno de los aspectos de la atención indagados.

Todos los aspectos metodológicos antes mencionados proporcionan evidencia suficiente de la validez de este estudio, lo cual hace posible la aplicación de técnicas inferenciales necesarias para la generalización de los resultados así obtenidos. Estos resultados mostraron coincidencias con varios de los estudios realizados en esta misma línea a nivel nacional. 
ACCESO Y SATISFACCIÓN CON SERVICIOS CURATIVOS: ANÁLISIS DE CASOS EN AFILIADOS AL RÉGIMEN CONTRIBUTIVO Y afiliados al rÉgimen subsidiado en un barRio estrato 2. Cali, Colombia

La principal limitación de esta investigación fue el no haber incluido en la metodología la consulta de fuentes primarias institucionales para el análisis de la oferta de servicios desde la perspectiva institucional, aunque sí se abordaron sus características desde la perspectiva de los usuarios. Ello hubiera complementado y enriquecido el análisis. La decisión se tomó por limitaciones presupuestales.

\section{Resultados}

La población general para la C16 caracterizada por grupos de edad, escolaridad y ocupación, muestra promedios aproximados a los reportados por el Censo 2005, típicos de una población urbana estrato 2. El 90,1\% de los hogares residían hacía más de un año en el BRI, esto muestra estabilidad y refuerza que las vivencias encontradas pertenecen al territorio.

La situación de afiliación al sGsss se comportó así: el 47,2\% de la población pertenecía al régimen contributivo, el 37,0\% al régimen subsidiado y un $1,6 \%$ se encontraba en el régimen de excepción o especial. El 11,6\% de las personas reportaron no estar afiliadas y un 2,6\% desconocían el tipo de afiliación. La comparación del aseguramiento de la C16, según los datos del 2006 de la Secretaría Municipal de Salud - proyectados a partir del Censo-, con el estudio en el BRI, mostró similitud en la afiliación al RC sumado el régimen de excepción (50,5\%), con diferencias en el Rs, para el cual la afiliación en el estudio fue 10 puntos porcentuales menor y en el reporte de las personas sin afiliación fue 8,9 puntos porcentuales más alto. Las diferencias en la proporción de personas afiliadas al Rs pueden explicarse por el proceso de desafiliación que el municipio tuvo en el 2008, dirigido a focalizar la cobertura sobre los niveles I y 2 del Sisben. Según la Secretaría de Salud Pública Municipal, en Cali se desafiliaron 30000 personas.
La prevalencia de los afiliados, tanto del RC como del Rs, que reportaron haber tenido un problema de salud o estar enfermos fue de $12,9 \%$ [11,3-14,8]; en el RC fue de $12,5 \%$ $[10,3-15,0]$ y en el Rs de $13,5 \%[1,1-16,4]$. Entre estos, la prevalencia de necesidad sentida de servicios curativos fue de $97,3 \%$ $[92,9-99,0]$ en el contributivo y $95,3 \%$, [88,3-98,2] en el subsidiado, con diferencias estadísticamente significativas $(p=0,022)$. La prevalencia de demanda expresada fue similar entre los regímenes, mientras que la prevalencia de demanda atendida fue mayor en el RC: 99,3\%. En concordancia con la puerta de entrada al Sistema, el 72,0\% de los afiliados que sintieron la necesidad de atención querían consultar al médico general. Se atendió al 97,8\% [94,1-99,2] de los afiliados que demandaron atención.

A nivel poblacional, los resultados del estudio mostraron que un $11,4 \%$ [9,97-12,83] de la población afiliada usó un servicio curativo. En el RC la población atendida fue similar al RS. Alrededor de una décima parte sintió la necesidad de consultar, demandó y usó los servicios curativos. En el RS se encontraron 2,2 puntos porcentuales menos entre los que sintieron un problema de salud y los que finalmente usaron el servicio.

Al relacionar los lugares donde consultaron los usuarios de servicios curativos con la red de prestación de servicios por régimen de afiliación, se encontró que hicieron uso de las instituciones adscritas a su red el 96,9\% de los afiliados al RC, en comparación con un $82,6 \%$ de los afiliados al Rs, diferencia estadísticamente significante $(\mathrm{p}<0,05)$.

Los problemas de salud ${ }^{4}$ por los cuales los usuarios consultaron a las IPS fueron enfer-

4 Se exploraron las siguientes variables: lesión por accidente o violencia; intoxicación o envenenamiento; mental, emocional o de los nervios; odontológico; atención del parto; atención quirúrgica; y otros 
medad general, en el $86,5 \%$ de los casos del contributivo, y en el subsidiado el $83,1 \%$; continúan en orden los problemas odontológicos (5,1\% RC y $11,3 \%$ Rs) y las lesiones por accidente o violencia (4,2\% RC y 4,7\% Rs). Mínimas frecuencias obtuvieron intoxicación o envenenamiento, enfermedad quirúrgica y problema mental, emocional o de los nervios. Los resultados de este estudio son similares a los hallazgos de la Encuesta Nacional de Salud (ENS) 2007.

El 50,0\% de las personas que consultaron por un problema de salud reportaron una reducción de sus actividades diarias o de su trabajo por este evento, siendo esta condición mayor en los afiliados al Rs $(64,4 \%)$. Este dato resulta similar al reportado por la ENDS 2005, según la cual en el país el 48,7\% de las personas redujeron sus actividades; la cifra para Cali, área metropolitana, en el mismo estudio fue del $45 \%$. La incapacidad total de realizar actividades por dicho problema de salud, no necesariamente otorgada por el proveedor de servicios, pero manifestada en sentirse gravemente enfermo, fue mayor entre los afiliados del RS ( $83 \%)$, siendo estadísticamente significante $(\mathrm{p}=0,003)$; en el RC fue del 63,3\%.

\section{Análisis de casos}

Vale la pena recordar que la información sobre consulta médica y los demás eventos específicos ${ }^{5}$ se recogió sobre las personas del estudio seleccionadas como casos según los criterios presentados en la metodología.

problemas de salud, dolor físico o malestar, diferentes a los anteriores. Algunas tomadas de Ministerio de la Protección Social, hoy Ministerio de Salud y Protección Social, Encuesta a Hogares Módulo II, en ENS 2007.

5 Consulta de especialistas, medicamentos, pruebas diagnósticas, hospitalización y atención en urgencias.
Características sociodemográficas de los casos. Entre los casos, el 35,5\% tenían entre 20 y 44 años, el 27,2\% entre 45 y 79 años y el $10,2 \%$ de 5 a 9 años; $57 \%$ eran mujeres. El $11,5 \%$ estaban en preescolar, $34,5 \%$ tenían estudios de primaria, 23,6\% secundaria, $5,8 \%$ tenían estudios técnicos/tecnológico y solo un $0,6 \%$ estudios universitarios; un $24,1 \%$ no tenía ninguna escolaridad. En ocupación, el 75,2\% fueron clasificados como población en edad de trabajar y el $42,4 \%$ como población económicamente inactiva (PEI). Dentro de la población económicamente activa (PEA), el 82,7\% se encontraban ocupados al momento de la encuesta y el 17,3 eran desempleados. Con relación a las posiciones ocupacionales de los encuestados, $54,9 \%$ eran obreros o empleados particulares, $39,4 \%$ trabajadores por cuenta propia, y el resto de posiciones reportaron menos del $5 \%$ en la distribución.

Afiliación y uso de IPs. Entre los casos, el $54,8 \%$ estaban afiliados al RC y el $45,2 \%$ al subsidiado. De los afiliados al RC, el 70,3\% eran beneficiarios y el $29,7 \%$ cotizantes.

En la figura 1 se georreferenciaron, en el RC y en el RS, según las direcciones exactas, las IPS consultadas por los casos y el nivel de concentración de las frecuencias de uso, lo cual permitió analizar el acceso geográfico, y se encontró una diferencia entre los dos regímenes en relación con la distancia al BRI.

Los casos reportaron pertenecer a 10 EPS en el RC y 6 en el RS. En el RC consultaron 32 IPS por servicios curativos, la mayoría de naturaleza privada; el 61,2\% del uso se distribuyó entre las 6 que ocuparon los primeros lugares, con proporciones entre el 15,5 y el 5,0\%. Entre los afiliados al Rs, fueron reportadas 15 IPs; el 70,9\% usó el hospital público de primer nivel, ubicado en el barrio, seguido de un $6,9 \%$ que usó el puesto de salud de un barrio contiguo. 


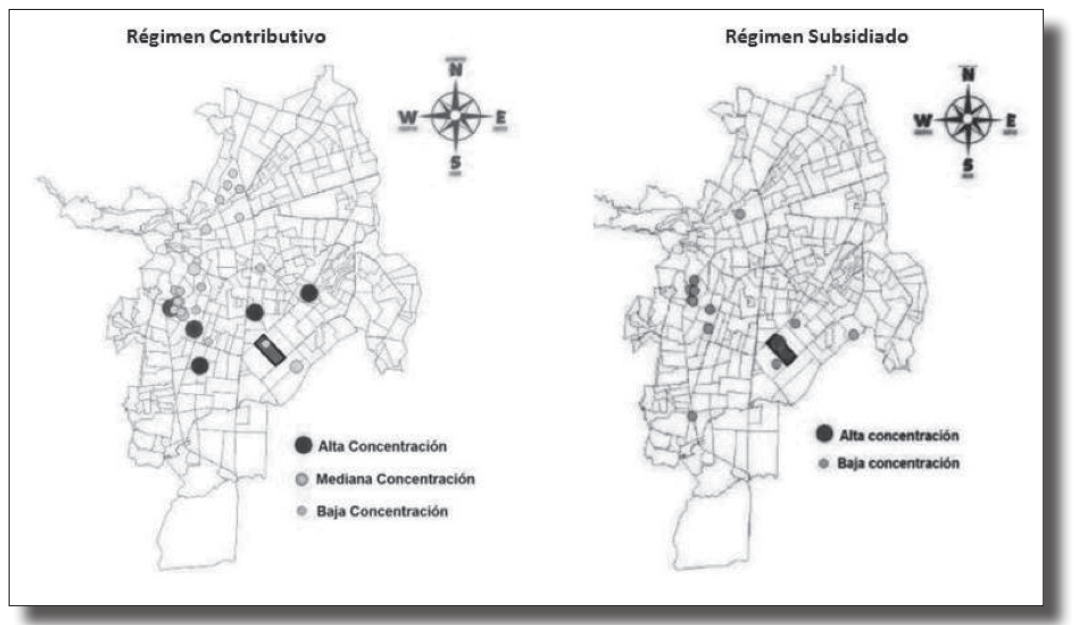

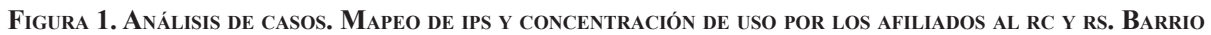
República de IsRael, 2009. CALI

Fuente: elaboración propia con datos del proyecto de investigación

En el Rs, todas las EPS ofrecen a su población como IPS de primer nivel las cercanas a su residencia que corresponden a la red de servicios de la ESE; las demás IPS usadas corresponden a otras ESE de nivel I, II y III y algunos prestadores privados, las cuales se ubican en diversos lugares de la ciudad, aunque la mayoría en el sector sur, concentradas en los barrios reconocidos por gran actividad en servicios de salud.

Se indagó la oportunidad de escoger la EPS y la IPS, así como la entrega escrita de información sobre derechos y servicios. La comparación entre ambos regímenes muestra mayores proporciones de oportunidad para escoger y recibir información en los casos afiliados al RC (ver tabla 1).

Acceso y satisfacción. Se indagó por la satisfacción con el servicio de consulta médica, y se encontró que los aspectos generadores de mayor insatisfacción en ambos regímenes son la duración de la consulta y la resolución del problema. Se hallaron diferencias significativas en el tiempo de espera en el momento de la consulta y en la ubicación del sitio (ver tabla 2).
Segunda consulta por el mismo problema de salud: el 17,1\% de los afiliados al RC y el $10,0 \%$ de los afiliados al Rs consultaron una segunda persona por el mismo problema de salud $(p=0,533)$. En ambos regímenes la principal razón para consultar a otra persona fue la insatisfacción: 70,6\% en el RC y 100\% en el Rs. Opciones de respuesta como "el problema no se resolvió", "no quedó satisfecho", "no confía en la primera persona que le atendió" y "otra persona se lo recomendó" fueron agrupadas en esta categoría. En el RC el segundo motivo para consultar una segunda persona fue la remisión.

El médico general fue la primera persona consultada por segunda vez entre los afiliados del RC (54,9\%); después de esta opción, se reportaron proveedores de medicina popular $(23,9 \%)$ y especialista $(16,8 \%)$, seguidos del odontólogo especialista $(4,4 \%)$. En los casos del Rs la primera persona fue el especialista (40\%), seguido del médico general $(29,1 \%)$, el médico alternativo $(22 \%)$ y el farmaceuta $(9,1 \%)$. El estudio cualitativo de Restrepo-Zea realizado en Medellín, sobre acceso a servicios de salud, análisis de barreras y estrategias de acceso reportó cómo 
Tabla 1. Reporte de los casos sobre libre escogencia e información en derechos en el SgSSs. Barrio República de IsRael, 2009. CALI

\begin{tabular}{|c|c|c|c|}
\hline \multirow{2}{*}{ Preguntas } & \multicolumn{2}{|c|}{ Afiliación al sGsss } & \multirow{2}{*}{ Valor de $\mathbf{p}^{*}$} \\
\hline & Contributivo (\%) & Subsidiado (\%) & \\
\hline \multicolumn{4}{|c|}{ Tuvo la oportunidad de escoger la entidad (EPS) } \\
\hline Sí & 78,4 & 36,5 & $\mathrm{p}=0,02$ \\
\hline No & 15,1 & 44,6 & \\
\hline Sin dato & 6,5 & 18,9 & \\
\hline \multicolumn{4}{|c|}{ Tuvo la oportunidad de escoger la institución (IPS) } \\
\hline Sí & 68,7 & 36,5 & $\mathrm{p}=0,02$ \\
\hline No & 24,7 & 44,6 & \\
\hline Sin dato & 6,6 & 18,9 & \\
\hline \multicolumn{4}{|c|}{ Le entregaron información escrita sobre sus derechos } \\
\hline $\mathrm{Si}$ & 50,0 & 38,1 & $\mathrm{p}=0,384$ \\
\hline No & 43,4 & 43,0 & \\
\hline Sin dato & 6,6 & 18,9 & \\
\hline \multicolumn{4}{|c|}{ Le informaron a qué servicios tenía derecho } \\
\hline Sí & 59,1 & 39,4 & $\mathrm{p}=0,81$ \\
\hline No & 34,3 & 41,7 & \\
\hline Sin dato & 6,6 & 18,9 & \\
\hline
\end{tabular}

$* \mathrm{p}<0,05$. Se excluyó la opción sin dato

Fuente: elaboración propia, cálculos del estudio

"varios de los entrevistados manifestaron que al no poder acceder a una cita, o por los largos períodos de espera, deciden muchas veces acudir al farmaceuta o a un tegua para que les venda algo que les pueda ayudar con su problema de salud, o para sostenerse mientras pueden acceder a la cita médica o con el especialista" (16).

El $40 \%$ de los afiliados al subsidiado consultaron un médico especialista como segunda opción, sin haber sido remitidos, lo cual implica consultas a particulares y gastos de bolsillo adicionales. Aunque el 30,9\% buscó la segunda atención en un hospital público (ESE), un 38,2\% reportó prestadores privados.

Entre los afiliados al RC, el centro médico 164 privado, la clínica privada o el médico de barrio, y el mismo lugar donde recibió la primera atención, fueron los lugares donde la mayoría $(73,7 \%)$ buscó el segundo proveedor de servicios; un $14 \%$ de los que buscaron una segunda consulta fueron atendido en casa; el 2,6\% consultó a una institución de la red pública de primer nivel.

Pruebas diagnósticas: al 51,1\% de los casos les ordenaron exámenes o pruebas diagnósticas, entre estos el $49 \%$ del RC y el $53,5 \%$ del Rs. Entre las pruebas ordenadas, el $66,5 \%$ fueron laboratorios (exámenes de sangre, orina, materia fecal, esputo, otras secreciones) y el 29,4\% imagenología (rayos $\mathrm{x}$, ecografías, mamografía, urografía, gammagrafía, cateterismos, resonancia, etc.). Entre los casos a quienes les ordenaron pruebas diagnósticas, el 79,8\% reportaron tomárselas todas, el 2,1\% solamente algunas y el 18,1\% ninguna. En el RC reportaron tomarse todas 
las pruebas un $85,7 \%$ y en el Rs el $73,2 \%$; se tomó alguna o ninguna prueba el $14,3 \%$ del RC y el 26,8\% del Rs; las diferencias entre estos valores reportados fueron estadísticamente significativas ( $\mathrm{p}<0,05)$. El 10,8\% de los usuarios del RC que se tomaron las pruebas, no reclamaron los resultados, mientras en el Rs fue el $1,3 \%$.

Las principales razones para no tomarse algunas o ninguna de las pruebas, fueron: $41,8 \%$ estaban esperando la fecha recomendada para tomárselas, $18,2 \%$ no tuvieron con qué pagar la cuota que les cobraban, y a un $15,4 \%$ se demoraron para darle la cita. En el RC las principales razones para no tomarse las pruebas fueron: esperar la fecha recomendada por el profesional de salud $(74,5 \%)$ y demora para darle la cita $(25,5 \%)$. En el RS los motivos más reportados fueron: no tener con qué pagar la cuota que le cobran $(29,5 \%)$, esperar la fecha $(23,1 \%)$, equipos dañados $(11,5 \%)$, descuido $(10,3 \%)$ y no se los autorizaron $(3,8 \%)$.

Las anteriores razones se consideraron barreras para acceder a los servicios (figura
2); las económicas, culturales y de acceso a información solo fueron reportadas por los casos del Rs, mientras que las administrativas se comportaron de manera similar en ambos regímenes. La categoría no aplica corresponde a la opción esperando la fecha recomendada por el profesional de salud. Las razones para no reclamar los resultados de las pruebas fueron: aún no le tocaba la cita 42,4\%, aún no estaban listos —estaba esperando la fecha indicada para reclamarlos$27,3 \%$, se los pasaron al médico $18,2 \%$ y no le entregaron los resultados a tiempo $12,1 \%$.

Con respecto a la satisfacción con los servicios de pruebas diagnósticas, la duración de la atención y el tiempo de espera fueron las mayores inconformidades en ambos regímenes. Los casos del RC se percibieron más insatisfechos con la información recibida, así como con la ubicación del sitio, el tiempo de espera y el tiempo de entrega de los resultados; los del Rs, con la duración de la atención, el trato recibido, la forma en que les hicieron el procedimiento y la comodidad; el tiempo de espera generó un poco más de insatisfacción en el RC (ver tabla 2).

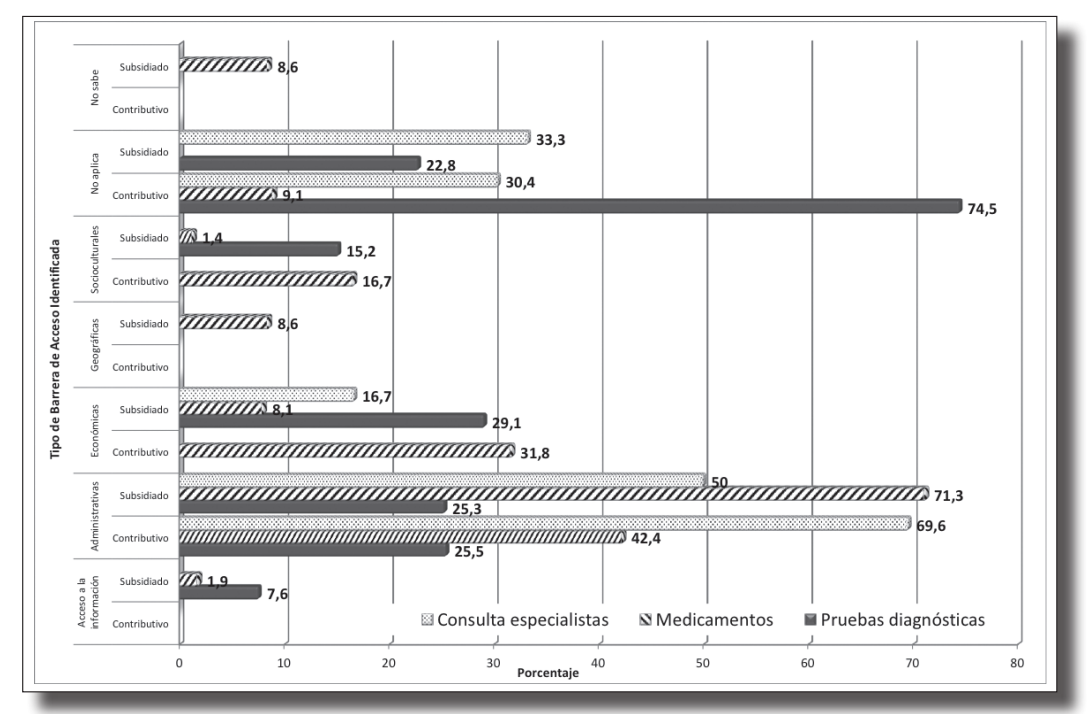

Figura 2. Barreras identificadas por régimen de afiliación. Barrio República de Israel, 2009. Cali Fuente: elaboración propia, cálculos del estudio 
Medicamentos: entre los casos que consultaron en el último mes, el $82,7 \%$ recibió una prescripción de medicamentos. Los reclamaron el $87,8 \%$ del RC y el 55,9\% del RS, diferencias estadísticamente significantes $(\mathrm{p}<0,05)$. En el RC reportaron mayores barreras económicas, geográficas y socioculturales, mientras en el Rs prevalecen las administrativas. La duración de la atención, la entrega de los medicamentos, la ubicación del sitio y el tiempo de espera generaron mayor insatisfacción. En la entrega de medicamentos hubo mayor insatisfacción entre los casos del Rs, 32,6\%, mientras en el RC fue de $18,9 \%$. Con relación a la ubicación del sitio, el Rs superó al RC en calificaciones desfavorables, con 23,1 y $16,5 \%$, respectivamente (ver tabla 2).

En concordancia con estos resultados, el estudio de Restrepo-Zea encontró cómo ante las dificultades en la continuidad, en cuanto a la entrega y el tipo de medicamentos formulados, los usuarios responden también con la compra particular y el "préstamo" de medicamentos entre quienes padecen enfermedades similares (16).

Aunque se revisó el estudio de Romero-Prada et al. (27), en el cual el análisis tuvo como único criterio de inclusión la selección de los medicamentos incluidos en el Acuerdo 29 del 2011 (actualización del Plan de Beneficios), quienes concluyen que la actualización permitió mejorar el acceso a muchos servicios de salud, estos resultados no son comparables metodológicamente.

Especialistas: un 24,4\% de los casos en ambos regímenes que consultaron en el último mes fueron remitidos al especialista, siendo el 22,6\% del re y el 26,5\% del Rs. Al indagar entre los no remitidos si consideraban que 166 su caso lo ameritaba, el 25,5\% del RC y el $23,7 \%$ del Rs respondieron afirmativamente.
Esta situación sugiere insatisfacción con el servicio recibido.

El estudio de Mejía (42) sobre equidad en el acceso a servicios de salud en Antioquia, con los datos de la Encuesta de Calidad de Vida 2003, reportó que $25 \%$ de los afiliados al RC y $14 \%$ del Rs fueron remitidos a un especialista. Resultados similares a los del presente estudio para el RC, mas no para el RS, donde se superan en 12,5 puntos lo encontrado en Antioquia.

Las especialidades más reportadas de remisión en el RC fueron: pediatría $28 \%$, cardiólogo $11,3 \%$ y ortopedista $10,7 \%$; en el Rs: ortopedista $26 \%$, odontólogo ${ }^{6} 21,2 \%$ y urólogo $15,1 \%$. Entre los remitidos consultaron, el 84,9\% del RC y el 75,3\% del Rs, sin diferencias estadísticamente significantes $(p=0,957)$. Las razones para no consultar en el RC fueron: la cita aún se encontraba pendiente presentó $30,4 \%$, la cita fue aplazada presentó $26,1 \%$ y la demora para dar las citas y negación de estas, ambos con un 21,7\%; mientras en el Rs fueron: el 33,3\% tenía la cita pendiente, $25 \%$ no le dieron la cita, $25 \%$ se demoran mucho para dar la cita y $16,7 \%$ no tenía con qué pagar la cuota. Las barreras para no consultarlos fueron en su mayoría administrativas; solo en el Rs se reportaron las económicas (ver figura 2).

En la consulta con especialistas se encontraron importantes porcentajes de insatisfacción en ambos regímenes, con relación a la resolución del problema (RC: $84,5 \%$ y Rs: $60 \%$ ) y la ubicación del sitio (RC: $58,1 \%$ y Rs: $37,6 \%$ ). En el RC el 43,3\% de los casos valoraron negativamente el tratamiento ordenado, el $31 \%$ la información recibida y el $35,7 \%$ la forma en que los examinaron. La comparación mostró diferencias impor-

6 Se releva la remisión al odontólogo, dado que fue reportado por los casos como especialista, aunque el odontólogo general en el sistema no se considera de esta manera. 
ACCESO Y SATISFACCIÓN CON SERVICIOS CURATIVOS: ANÁLISIS DE CASOS EN AFILIADOS AL RÉGIMEN CONTRIBUTIVO Y afiliados al régimen subsidiado en un barrio estrato 2. Cali, Colombia

Tabla 2. Resumen de satisfacción CON las Características del SERVicio de CONSUlta, PRuebas diagnósticas y entrega de medicamentos por RÉgimen. Barrio República de Israel, 2009. CALi

\begin{tabular}{|c|c|c|c|c|c|c|c|c|c|}
\hline \multirow[b]{2}{*}{ Categoría } & \multicolumn{3}{|c|}{$\begin{array}{l}\text { Valoración de la atención en } \\
\text { el servicio de consulta }\end{array}$} & \multicolumn{3}{|c|}{$\begin{array}{c}\text { Valoración de la atención } \\
\text { recibida en servicios de } \\
\text { pruebas diagnósticas }\end{array}$} & \multicolumn{3}{|c|}{$\begin{array}{l}\text { Valoración de la atención en } \\
\text { entrega de medicamentos }\end{array}$} \\
\hline & $\begin{array}{l}\text { Contri- } \\
\text { butivo } \\
(\%)\end{array}$ & $\begin{array}{l}\text { Subsi- } \\
\text { diado } \\
(\%)\end{array}$ & $p$ & $\begin{array}{l}\text { Contri- } \\
\text { butivo } \\
(\%)\end{array}$ & $\begin{array}{c}\text { Subsi- } \\
\text { diado } \\
(\%)\end{array}$ & $p$ & $\begin{array}{l}\text { Contri- } \\
\text { butivo } \\
(\%)\end{array}$ & $\begin{array}{l}\text { Subsi- } \\
\text { diado } \\
(\%)\end{array}$ & $p$ \\
\hline \multicolumn{10}{|c|}{ Tiempo de espera } \\
\hline Satisfecho & 75,3 & 57,2 & \multirow{2}{*}{0,01} & 64,6 & 67,4 & 0,83 & 82,5 & 83,8 & 0,78 \\
\hline Insatisfecho & 24,7 & 42,8 & & 35,4 & 32,6 & & 17,5 & 16,2 & \\
\hline \multicolumn{10}{|c|}{ Duración de la atención } \\
\hline Satisfecho & 24,7 & 62,1 & \multirow{2}{*}{0,77} & 78,9 & 69 & 0,14 & 39,3 & 43,8 & 0,18 \\
\hline Insatisfecho & 38,3 & 37,9 & & 21,1 & 31 & & 69,7 & 56,2 & \\
\hline \multicolumn{10}{|c|}{ Trato recibido } \\
\hline Satisfecho & 86,8 & 87,1 & \multirow{2}{*}{0,66} & 98,6 & 94,8 & 0,46 & 89,4 & 96,2 & 0,82 \\
\hline Insatisfecho & 13,2 & 12,9 & & 1,4 & 5,2 & & 10,6 & 3,8 & \\
\hline \multicolumn{10}{|c|}{ Información recibida } \\
\hline Satisfecho & 82,1 & 87,6 & \multirow{2}{*}{0,93} & 74,0 & 93,0 & 0,41 & 84,4 & 93,1 & 0,50 \\
\hline Insatisfecho & 17,9 & 12,4 & & 26,0 & 7 & & 15,6 & 6,9 & \\
\hline \multicolumn{10}{|c|}{ Ubicación del sitio } \\
\hline Satisfecho & 74,3 & 90,9 & \multirow{2}{*}{0,002} & 69,0 & 83,8 & 0,07 & 83,5 & 76,9 & 0,77 \\
\hline \multirow[t]{2}{*}{ Insatisfecho } & 25,7 & 9,1 & & 31,0 & 16,2 & & 16,5 & 23,1 & \\
\hline & \multicolumn{3}{|c|}{ Resolución del problema } & \multicolumn{3}{|c|}{$\begin{array}{l}\text { Tiempo de entrega de resul- } \\
\text { tados }\end{array}$} & \multicolumn{3}{|c|}{ Entrega de medicamentos } \\
\hline Satisfecho & 56,4 & 47,2 & \multirow{2}{*}{0,36} & 73,2 & 87,8 & 0,26 & 81,1 & 67,4 & 0,09 \\
\hline \multirow[t]{2}{*}{ Insatisfecho } & 43,6 & 52,8 & & 26,8 & 12,2 & & 18,9 & 32,6 & \\
\hline & \multicolumn{6}{|c|}{ Comodidad } & & & \\
\hline Satisfecho & 88,7 & 82,4 & \multirow{2}{*}{0,06} & 98,2 & 86,9 & 0,11 & & & \\
\hline \multirow[t]{2}{*}{ Insatisfecho } & 11,3 & 17,6 & & 1,8 & 13,1 & & & & \\
\hline & \multicolumn{3}{|c|}{ Forma en que lo examinaron } & \multicolumn{3}{|c|}{$\begin{array}{c}\text { Forma en que le hicieron el } \\
\text { procedimiento }\end{array}$} & & & \\
\hline Satisfecho & 84,7 & 85,5 & \multirow{2}{*}{0,62} & 96,8 & 93,0 & 0,51 & & & \\
\hline \multirow[t]{2}{*}{ Insatisfecho } & 15,3 & 14,5 & & 3,2 & 7 & & & & \\
\hline & \multicolumn{3}{|c|}{ Tratamiento ordenado } & & & & & & \\
\hline Satisfecho & 74,5 & 84,4 & \multirow{2}{*}{0,32} & & & & & & \\
\hline Insatisfecho & 25,5 & 15,6 & & & & & & & \\
\hline
\end{tabular}

Fuente: elaboración propia, cálculos del estudio 
tantes, con significancia estadística, en el tiempo para ser atendido en sala de espera y la comodidad, afectando en ambos casos mucho más al Rs (ver tabla 3).

Hospitalización: al 14,4\% de los casos, les ordenaron una hospitalización en el último año, distribuidos el 9,7\% en el RC y el 20,0\% en el Rs, diferencias estadísticamente significantes $(p=0,024)$. Entre los que tuvieron una orden de hospitalización fueron internados, al menos por una noche, el 90,8\% del RC y el 88,3\% del Rs. La ENDS 2005 reportó para el país un $5 \%$ de personas hospitalizadas en los últimos 12 meses; en Cali, área metropolitana se encontró un 4,8\%. En el presente estudio los resultados duplican los de la ENDS.

La principal causa de hospitalización ${ }^{7}$ en ambos regímenes fue la enfermedad general, encontrándose una mayor proporción en los casos del Rs $(77,6 \%)$ con relación al RC $(48,3 \%)$. En segundo lugar, la cirugía programada con un $12 \%$, a la que le siguen los accidentes y la violencia con $10,1 \%$, procedimientos diagnósticos especializados $8,20 \%$ y enfermedad mental $3,2 \%$. No se reportaron causas obstétricas. Las opciones cirugía programada y accidente fueron reportadas en mayor porcentaje por los casos del RC, mientras en el Rs fueron los procedimientos diagnósticos especializados y la enfermedad mental.

Para el 71,2\% de los casos del re y el 38,8\% del Rs se resolvió el problema de salud por el cual fue hospitalizado; resolución parcial y no resolución fue reportado por el $28,8 \%$ del rc y el 61,2\% del Rs. Con respecto a la satisfacción con el servicio de hospitalización, en ambos regímenes se reportaron porcentajes altos de satisfacción con el profesional

7 Las opciones de respuesta para esta pregunta se definieron a partir de la Encuesta Nacional de Demografía y Salud 2005 y se ajustaron de acuerdo con los resultados de la prueba piloto. médico; en el Rs se manifestó insatisfacción con: tiempo de espera para ser hospitalizado $(37,1 \%)$, duración de la hospitalización $(36,7 \%)$ y privacidad $(33,7 \%)$, mientras que en el RC se valoró negativamente la atención por el personal de enfermería $(31,7 \%)$, el trato recibido $(30,7 \%)$ y la resolución del problema $(28,8 \%)$.

Urgencias: entre todos los casos, el 49,6\% del RC y el 38,7\% del Rs sintieron o tuvieron la necesidad de usar el servicio de urgencias en el último año; el $100 \%$ de estos fueron atendidos. En la ENs 2007, de los usuarios atendidos en servicios de urgencias, el $49 \%$ correspondían al RC y el $35 \%$ al subsidiado, cifras similares a las de este estudio.

El tiempo de espera entre el momento en que los casos entraron al servicio de urgencias y el momento en que el médico les atendió fue menor de 1 hora para el $45,7 \%$, entre 1 y 2 horas para el 32,9\%, 3 y 5 horas para el $14,4 \%$, seis horas y más para el $2,6 \%$, y no recordó el tiempo de espera el 4,3\%. Por regímenes, se observa cómo el 51,8\% del RC y el 36,2\% del Rs esperó menos de una hora para ser atendido; de una a dos horas esperó un $26,8 \%$ del RC y un $42,3 \%$ del Rs; entre 3 y 5 horas fueron reportadas por un $17,8 \%$ del RC, contra un 9,4\% del Rs; 6 y más horas de espera se reportaron en un 6,6\% del Rs. Las esperas mayores a 3 horas podrían corresponder, más que a demandas de urgencias, a consultas prioritarias de salud.

Con relación a la satisfacción con el servicio de urgencias se encontraron percepciones negativas importantes en el RC y en el RS, para: resolución del problema $(43,8$ y $53,6 \%)$, tiempo de espera para ser atendido por el médico $(43,5$ y $53,5 \%)$ y duración de la atención (30,7 y 42,7\%). Entre los casos de RS se obtuvieron porcentajes más altos para valoraciones negativas sobre comodidades, información recibida, privacidad, duración 
de la atención y tiempo para ser atendido. En el RC solo el tiempo para ser atendido por el médico tuvo una valoración negativa superior a la del RS. Se encontró diferencia significativa en la privacidad, donde el 30,2\% del RC la valoró negativamente, contra el $63,2 \%$ del Rs (ver tabla 3 ).

Gastos de bolsillo: se indagaron para cada servicio de salud atendido y se encontró que un porcentaje importante tuvieron que pagar (ver tabla 4). Con excepción de la hospitalización, el valor moda pagado en cada tipo de servicio fue de $\$ 2000$, lo cual significa que muchos de los usuarios pagaron la cuota moderadora mínima establecida para el RC en el momento de la indagación. Esto es coherente con el estrato socioeconómico del BRI. La mayoría de estos pagos están reglamentados en el Sistema, pero varios de los casos reportaron haber realizado pagos por fuera de este; por ejemplo, a médicos particulares, medicinas alternativas o sistema popular de salud. El hallazgo de pagos en el servicio de urgencias se podría atribuir a una clasificación de la demanda como consulta prioritaria.

\section{Discusión y conclusiones}

Si bien desde que se realizó la investigación y hasta la fecha, en Colombia el sGsss ha tenido transformaciones dirigidas a mejorar el acceso y lograr igualdad en los planes de beneficios, los problemas de acceso real a los servicios, como los encontrados en este estudio se mantienen, lo cual se evidencia en el informe de la Defensoría del Pueblo del 2014 (3) y en la exposición de motivos de la Ley Estatutaria, expedida para proteger el derecho fundamental a la salud y sancionada en febrero del 2015 (12).

GarcíaSubirats et al., en sus publicaciones sobre inequidades en el acceso al cuidado continuo y comparación de factores que influyen en el acceso en dos municipios de Brasil y dos de Colombia, concluyen que en Colombia, a pesar de múltiples ajustes al SGsss, persisten las inequidades, lo que favorece especialmente a individuos de clases sociales más altas (14). Sobre las barreras de acceso evidenciaron importantes obstáculos en el acceso inicial a la atención y en la resolución del problema. Las mayores barreras geográficas y económicas y la necesidad de autorización de las aseguradoras constituyeron los factores más relevantes, aunque también encontraron diferencias por nivel educativo y tipo de aseguramiento; las barreras parecen estar relacionadas con la naturaleza segmentada del sistema colombiano, no universal, y por la participación de las compañías de seguros (15). Muchos de los resultados de estos estudios coinciden con los de la anterior publicación sobre servicios preventivos (13).

Dado que en el modelo de Aday y Andersen, los componentes están fuertemente interrelacionados, esta discusión integra y conecta las diversas variables en un esfuerzo por presentar la complejidad del fenómeno. También se retoman los planteamientos de Frenk (34) en su propuesta sobre medición de accesibilidad. Es así como a nivel teórico se plantea que ante una necesidad de atención en salud el individuo desea y busca dicha atención (34); sin embargo, hay situaciones subjetivas de la población y objetivas de la oferta que influyen en esta cadena de eventos; es decir, que percibir un problema de salud, sentir la necesidad y buscar la atención en un servicio curativo depende de diversos factores como los económicos, los sociales y los culturales de la población, entre otros, y de distintos factores de la oferta de servicios, en lo cual coinciden la mayoría de los estudios referenciados. 


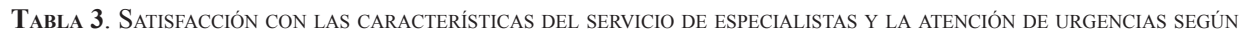
RÉGIMEN. BARRIo REPÚBLICA de ISRAel, 2009. CALI

\begin{tabular}{|c|c|c|c|c|c|c|}
\hline \multirow[b]{2}{*}{ Categoría } & \multicolumn{3}{|c|}{ Valoración de la atención por especialista } & \multicolumn{3}{|c|}{ Valoración de la atención en urgencias } \\
\hline & $\begin{array}{c}\text { Contributivo } \\
(\%)\end{array}$ & $\begin{array}{l}\text { Subsidiado } \\
(\%)\end{array}$ & $\mathbf{p}$ & $\begin{array}{c}\text { Contributivo } \\
(\%)\end{array}$ & $\begin{array}{l}\text { Subsidiado } \\
(\%)\end{array}$ & $\mathbf{p}$ \\
\hline & \multicolumn{3}{|c|}{$\begin{array}{c}\text { Tiempo transcurrido entre la orden médica } \\
\text { y el día en que le atendió }\end{array}$} & \multicolumn{3}{|c|}{$\begin{array}{l}\text { Tiempo de espera para ser atendido por } \\
\text { el médico }\end{array}$} \\
\hline Satisfecho & 79,7 & 74,5 & \multirow{2}{*}{0,45} & 56,5 & 46,5 & \multirow{2}{*}{0,29} \\
\hline \multirow[t]{2}{*}{ Insatisfecho } & 20,3 & 25,5 & & 43,5 & 53,5 & \\
\hline & \multicolumn{3}{|c|}{$\begin{array}{c}\text { Tiempo de espera para ser atendido (sala } \\
\text { de espera) }\end{array}$} & \multicolumn{3}{|c|}{ Duración de la atención } \\
\hline Satisfecho & 94,5 & 68,2 & \multirow{2}{*}{0,01} & 69,3 & 58,3 & \multirow{2}{*}{0,31} \\
\hline \multirow[t]{2}{*}{ Insatisfecho } & 5,5 & 31,8 & & 30,7 & 42,7 & \\
\hline & \multicolumn{6}{|c|}{ Trato recibido } \\
\hline Satisfecho & 89,9 & 91,7 & \multirow{2}{*}{0,65} & 83,1 & 68,6 & \multirow{2}{*}{0,19} \\
\hline \multirow[t]{2}{*}{ Insatisfecho } & 10,1 & 8,3 & & 16,9 & 31,4 & \\
\hline & \multicolumn{3}{|c|}{ Forma en que lo examinaron } & \multicolumn{3}{|c|}{ Atención del profesional médico } \\
\hline Satisfecho & 64,3 & 87,1 & \multirow{2}{*}{0,50} & 81,6 & 78,3 & \multirow{2}{*}{0,21} \\
\hline \multirow[t]{2}{*}{ Insatisfecho } & 35,7 & 12,9 & & 18,4 & 21,7 & \\
\hline & \multicolumn{3}{|c|}{ Comodidad } & \multicolumn{3}{|c|}{$\begin{array}{l}\text { Atención profesional del personal de } \\
\text { enfermería }\end{array}$} \\
\hline Satisfecho & 100 & 77,9 & \multirow{2}{*}{0,01} & 96 & 80,6 & \multirow{2}{*}{0,74} \\
\hline \multirow[t]{2}{*}{ Insatisfecho } & 0,0 & 22,1 & & 4 & 19,4 & \\
\hline & \multicolumn{6}{|c|}{ Información recibida } \\
\hline Satisfecho & 69,0 & 80,7 & \multirow{2}{*}{0,8} & 77,9 & 61,4 & \multirow{2}{*}{0,18} \\
\hline \multirow[t]{2}{*}{ Insatisfecho } & 31,0 & 19,3 & & 22,1 & 38,6 & \\
\hline & Trata & to ordenado & & & vacidad & \\
\hline Satisfecho & 56,7 & 88 & 089 & 69,8 & 36,8 & 0.03 \\
\hline Insatisfecho & 43,4 & 12 & 0,09 & 30,2 & 63,2 & , \\
\hline & & ón del sitio & & & odidades & \\
\hline Satisfecho & 41,9 & 62,4 & 056 & 75,2 & 54,4 & 0,40 \\
\hline Insatisfecho & 58,1 & 37,6 & 0,50 & 24,8 & 45,6 & $0,+0$ \\
\hline & & & lución a & l problema & & \\
\hline Satisfecho & 15,5 & 40,0 & 026 & 56,2 & 43 & 006 \\
\hline Insatisfecho & 84,5 & 60 & 0,20 & 43,8 & 53 & 0,00 \\
\hline
\end{tabular}

Fuente: elaboración propia, cálculos del estudio 


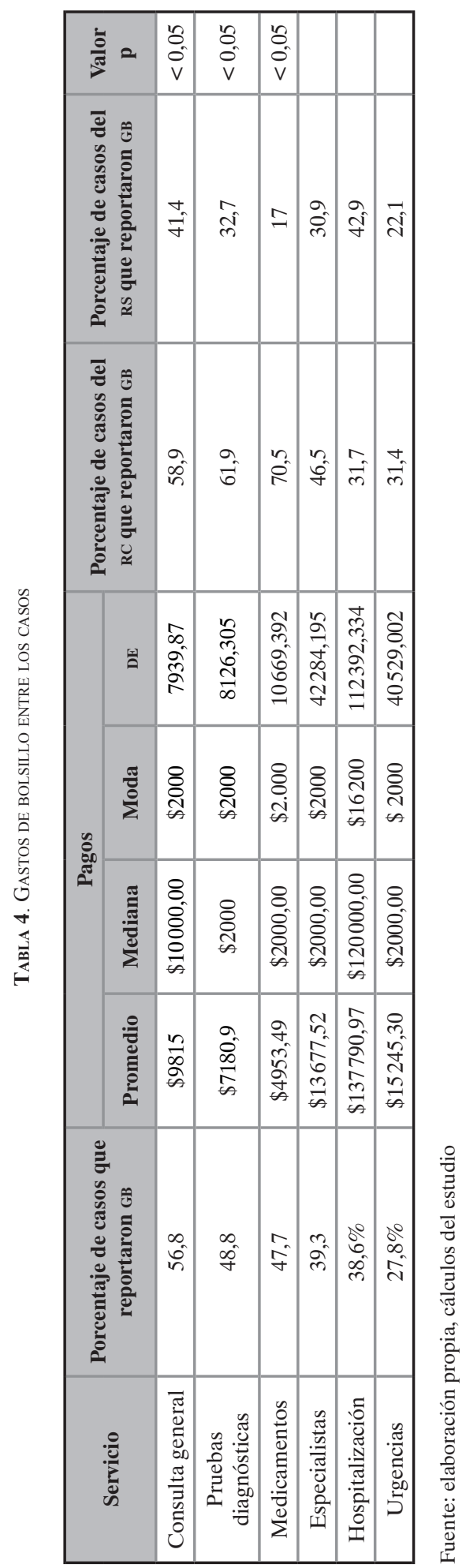


Acceso potencial: características de la población

Para Aday y Andersen, las características de predisposición al acceso observadas en la población, los recursos disponibles en la comunidad y las necesidades percibidas por los "consumidores" y evaluadas por los prestadores de servicios determinan en parte el acceso potencial a los servicios.

En el estudio se encontraron características diferentes, en un mismo estrato, en variables socioeconómicas entre el grupo afiliado al RS y el afiliado al RC, y aunque son similares en cuanto a estructura demográfica, presentan discrepancias en escolaridad y ocupación que reflejan mejores condiciones para la población del RC.

La similitud de las características demográficas entre los grupos comparados, específicamente en estructura de edad y sexo, marca eventos de riesgo compartidos por ciclos de vida y grupos específicos de población; sin embargo, estos se ven influidos por las condiciones del contexto en que transcurre la vida de las comunidades. Por ejemplo, si se revisan las causas de consulta general, de hospitalización y de urgencias, la enfermedad general es la primera causa, con leves diferencias entre los regímenes dependiendo del servicio utilizado; los casos del RC reportan más accidentes, probablemente por los riesgos laborales o los propios del desplazamiento ligado a la ocupación; la violencia y la enfermedad mental se reportan en ambos grupos y son expresiones propias del contexto social donde convive la población.

Según Frenk, parte del poder de utilización de los servicios se soporta en los recursos disponibles en la comunidad (34), y en este sentido es una ventaja haber logrado cober-

172 turas por encima del $90 \%$ de afiliación al SGSSs, lo que se constituye en un importante activo de la comunidad para el acceso a la atención. Dicha situación se refleja en estos resultados, dado que el $92 \%$ de los afiliados al Rs y el $96 \%$ del RC que sintieron la necesidad de consultar un servicio curativo por un problema de salud, solicitaron atención.

En lo relacionado con la información impresa sobre los derechos en salud y el portafolio de servicios que deben conocer los afiliados para ejercer estos derechos, se encontró diferencia entre los grupos, resultando más favorable para el RC, aunque los hallazgos son preocupantes para ambos regímenes. Estos resultados coinciden con Varela, Carrasquilla, Tono y Samper (43), como también lo evidencian Restrepo et al. (16), y se constituyen en obstáculos en ambos grupos estudiados, aunque más acentuados en el Rs para interactuar en el sistema y para el acceso a los servicios.

Como recursos también se incluyen los propios del desplazamiento hacia las instituciones de salud, condicionados por la ubicación de los prestadores y de los puntos de atención al usuario de las EPs donde se autorizan las actividades de prestación. Los hallazgos del estudio muestran mejores condiciones de acceso en términos de distancia para los afiliados al Rs. En general, los prestadores del RC están mucho más dispersos y distantes del barrio, sin embargo, las pocas prestadoras privadas más cercanas se usan con mayor frecuencia, lo que sugiere que los usuarios del RC valoran positivamente la cercanía.

Rubio (44) encontró que en Bogotá un 30\% de la población usa solamente recursos propios para resolver necesidades de salud, en esta proporción participan, en primer lugar, la población no afiliada, en segundo lugar la subsidiada y por último la contributiva (44). En concordancia con ello, en este estudio, al analizar el uso de la red de prestadores a los que pertenecían los casos, se encontró 
ACCESO Y SATISFACCIÓN CON SERVICIOS CURATIVOS: ANÁLISIS DE CASOS EN AFILIADOS AL RÉGIMEN CONTRIBUTIVO Y afiliados al rÉgimen subsidiado en un barRio estrato 2. Cali, Colombia

cómo el 18\% del Rs hizo uso de otras instituciones, con diferencias estadísticamente significantes $(\mathrm{p}<0,05)$ con respecto al $\mathrm{RC}$, lo cual implica gastos de bolsillo adicionales en salud. Los afiliados al RC deben sumar en sus gastos, además de las cuotas moderadoras y los copagos, los costos del desplazamiento hacia y desde las redes de prestación.

Desde la propuesta de Frenk, se podría concluir que la población del RC, por su condición de contar al menos con una persona en los hogares con vinculación formal al sistema laboral, tendría opción de contrarrestar los obstáculos o resistencias de los servicios e imponer su poder de utilización, conferido por el salario, sin embargo, en esta población prima el salario mínimo legal colombiano, sobre el cual los gastos de bolsillo en salud representan cerca del $6 \%$ del ingreso de las personas más pobres (45).

Estos hallazgos muestran obstáculos como los descritos por Frenk, los cuales parecen estar transformando el sentido y los significados de la necesidad de atención en la población de ambos regímenes; las prevalencias de problemas de salud percibidos y el uso de servicios curativos en el último mes (12,9 y $11,4 \%$, respectivamente) fueron relativamente bajas para una población pobre, que reportó un $14 \%$ de percepción desfavorable sobre su estado de salud y una prevalencia de $11 \%$ de enfermedades crónicas. En el mismo sentido, el estudio de Rubio (44) mostró una de las más bajas prevalencia de necesidad sentida de enfermedad en el país en población pobre, estimada en el 9,2\% (44).

\section{Acceso potencial: características de la oferta}

Según Aday y Andersen, las características de la oferta incluyen la disponibilidad de los servicios y su organización, pues estos deben estar disponibles en el momento y lugar en que el paciente los necesita y su forma de ingreso al sistema debe ser clara. En este estudio se exploraron las características de la oferta desde la perspectiva de la población, a través de la percepción manifestada por los casos.

Con relación a los prestadores de servicios, en el RC la mayoría de las IPs consultadas con mayor frecuencia pertenecían a las EPS o tenían sociedades importantes con estas -integración vertical-, lo que afecta su independencia administrativa y tiende a favorecer los intereses de la entidad y no los de los usuarios. En el Rs, la red de la ESE de nivel I era y sigue siendo monopólica en el barrio y las opciones de otras redes son mínimas, dado que por norma las EPS-S deben contratar al menos el $60 \%$ con prestadores públicos; por consiguiente, tanto desde las EPS como desde los prestadores, el poder está centrado en la institución y no en el usuario, y con ello se afecta el poder de utilización o acceso y aumenta la resistencia, es decir, los obstáculos a la búsqueda y obtención de la atención, como los denomina Frenk (34).

Como se discutió anteriormente en las características de la población sobre el acceso potencial, "la ubicación de la oferta es una de las diferencias más notables entre los regímenes, constituyéndose en obstáculo ecológico o barrera geográfica para una importante proporción de los afiliados al RC. Mientras la red básica de servicios del RS está ubicada dentro del barrio o a pocas cuadras del mismo en sectores aledaños, disminuyendo gastos de transporte y tiempo de desplazamiento en la mayoría de los usuarios, las redes de las EPS-C están por fuera del barrio (13, p. 171).

Las razones para no demandar el servicio y para no ser atendido son similares entre regímenes en cuanto a barreras administrativas, no así los obstáculos financieros o las barreras económicas, que fueron mucho más 
frecuentes en el Rs. Esta situación podría explicarse, por un lado, por los gastos en tiempo y transporte, y por otro lado, por las cuotas moderadoras y copagos, los cuales continúan, a pesar de que la Corte Constitucional —Sentencia T760 del 2008- ordenó que estos no podían obstaculizar el acceso a los servicios de salud, dado que pueden generar postergación de la consulta, aspecto que podría estar relacionado con los hallazgos de este estudio, donde se muestra gravedad de los problemas de salud (sentirse gravemente enfermo) en una proporción importante de los casos que consultaron.

La ENS 2007 encontró que los proveedores de servicios están dando a la mayoría de los usuarios información durante la atención, con similitud en ambos regímenes, sin embargo, esta investigación profundizó en el contenido de la información y encontró que esta no es completa; casi un $50 \%$ de los casos no fueron informados sobre su tratamiento y porcentajes mayores no recibieron información sobre cuidados que seguir o sobre su diagnóstico.

\section{Acceso realizado: utilización de servicios de salud}

Debido a que no siempre todos los individuos que desean ingresar al sistema lo logran, es necesario aplicar un tipo de validación externa, que indique si las características de la población y las de la oferta determinan una diferencia en la obtención de la atención. A este efecto Aday y Andersen proponen examinar factores propios de la utilización de los servicios de atención, en poblaciones específicas en el transcurso del tiempo. En el presente estudio las variables de utilización se exploraron en la población y no en los prestadores de servicios de salud. de la población del barrio afiliada al RC y al
RS que demandó un servicio curativo fue atendida en el primer nivel de atención en la consulta médica general; a este respecto, en Bogotá, Rubio (44) encontró entre quienes tuvieron un problema de salud, cómo el $81,6 \%$ de la población del RC y el 73,2\% del RS accede a los servicios de salud (44). Sin embargo, al explorar en los casos sobre la integralidad de la atención se observaron barreras para ser atendidos por especialistas y para la toma de pruebas diagnósticas y entrega de medicamentos.

Según lo anterior, los resultados en el BRI mostraron una cifra muy positiva para quienes demandaron atención en el primer contacto con el sistema de salud, pero la situación cambió cuando se estimó el uso de los servicios con relación al tamaño de la población, pues las prevalencias de percepción de un problema de salud y uso de servicios curativos en el último mes fueron relativamente bajas, para ser esta una población pobre con alta prevalencia de enfermedades crónicas y un perfil demográfico en transición, ya que alrededor de una décima parte de la población cumplió secuencialmente tres condiciones: 1) sintió la necesidad de consultar un servicio curativo, 2) demandó y 3) uso los servicios.

En el BRI, al igual que en todo el país, los afiliados al RC y los afiliados al Rs financian sus gastos en salud a través del aseguramiento, en la medida en que demandan la atención y la cobertura del pos los incluye. Sin embargo, los copagos, las cuotas de recuperación, los servicios no cubiertos y la búsqueda de atención por fuera de los prestadores asignados por las EPS generaron gastos de bolsillo tanto para la población afiliada al RC como para la del Rs, con una carga superior para los usuarios afiliados al RC; la única excepción fue el servicio de hospitalización. De igual manera, se observó en ambos regímenes que algunos 
ACCESO Y SATISFACCIÓN CON SERVICIOS CURATIVOS: ANÁLISIS DE CASOS EN AFILIADOS AL RÉGIMEN CONTRIBUTIVO Y afiliados al rÉgimen subsidiado en un barRio estrato 2. Cali, Colombia

hogares se vieron obligados a incurrir en pagos que superaban su capacidad económica.

Estos resultados se correlacionan con los hallazgos de los siguientes trabajos: por un lado, con los del estudio de hogares realizado en Cartagena sobre gasto privado en salud, donde se encontró que las familias que pertenecen a los estratos socioeconómicos más bajos ( 1 o 2$)$ incurren en un gasto de bolsillo mayor con relación a su ingreso que los estratos más altos (46), y por otro lado, con los hallazgos del estudio cualitativo de Garcés-Palacio, realizado en Medellín, donde se identificaron barreras y facilitadores del sistema de salud relacionados con el seguimiento de anormalidades citológicas en mujeres de bajos ingresos usuarias de la red pública, y además se encontró notoria la categoría de barreras económicas, dado que "las participantes señalaron reiteradamente que tuvieron que destinar dinero para pagar pasajes, citologías, otros exámenes diagnósticos, consultas con médicos generales y especialistas, medicamentos, tratamientos y copagos. Incluso, varias mujeres tuvieron que dejar su trabajo para destinar tiempo a tramitar autorizaciones, lo que acentuó su vulnerabilidad económica" (26). Todo lo anterior muestra una clara situación de inequidad vertical en el financiamiento del sistema de salud.

\section{Acceso realizado: satisfacción del usuario}

Andersen también incluye la subjetividad de los usuarios de los servicios dentro de las evaluaciones, al medir la conformidad o el descontento de los individuos por obtener un servicio de salud, mientras que Freeborn y Greenlick, citados por Andersen (30), proponen basarse en la opinión de los pacientes sobre la disponibilidad de los servicios en el momento y el lugar requeridos y por la mejoría que perciben como resultado de la atención recibida.
Los hallazgos evidencian que los casos experimentaron una insatisfacción generalizada respecto a algunas características de la atención recibida en los servicios curativos, y también en cuanto a la resolución de los problemas por los cuales consultaron, con diferencias significativas entre los regímenes, según el tipo de servicio y la variable calificada. Estos hallazgos son similares a los reportados por Garcia-Subirats et al. (15), quienes luego de comparar Colombia y Brasil, muestran que la proporción de personas que reportaron no tener resuelto su problema de salud fue mayor en Colombia para todos los tipos de atención, con una diferencia significativa en el nivel primario de atención y en urgencias. La resolución en el Rs fue significativamente menor que en el RC. Las principales razones reportadas en Colombia por los usuarios sobre la no resolución de los problemas fueron: que no hubo ninguna mejora $(29,9 \%)$, que las pruebas no se pudieron llevar a cabo (10\%), que los medicamentos no funcionaron $(8,4 \%)$ y que no lograron conseguir una cita con el especialista $(8,4 \%)(15)$. En el estudio del BRI, un porcentaje considerable de los casos reportaron no resolución de sus problemas de salud después de consultar tanto médicos generales $(43,6 \% \mathrm{RC}, 52,8 \% \mathrm{RS})$ como especialistas ( $84,5 \%$ RC, $60,0 \%$ Rs) e inconformidades con las características de los servicios (ver tablas 2 y 3 ).

Estos hallazgos riñen en general con todo lo normado en el sistema de garantía de calidad, aunque deben alarmar especialmente a la red pública, la cual parece aportar más a la inequidad entre los regímenes. Esto quizá implique revisar los criterios con los cuales las aseguradoras seleccionan sus redes de prestación de servicio y mejorar las acciones de vigilancia y control de las autoridades sanitarias.

Del estudio en el BRI se destaca cómo entre quienes tuvieron un problema de salud, la 
prevalencia de necesidad sentida de servicios curativos estuvo por encima del $95 \%$ en ambos regímenes, la prevalencia de demanda expresada fue similar, y entre los que buscaron atención, fueron atendidos el 97,8\%. El hecho de que la mayoría de la población refiera ser atendida en el primer nivel, es una franca ganancia del sGsss y podría traducirse en beneficios importantes si se lograra integralidad en la atención, pero los hallazgos dieron cuenta de la existencia de múltiples factores que se constituyen en barreras para lograr acceso equitativo y con calidad, que se reflejan en los innumerables fallos de sentencias de la Corte Constitucional y en los informes de las entidades de control social y administrativo como la Procuraduría, la Personería o la Contraloría.

La escasa disponibilidad de recursos en una población pobre como la de este estudio afecta el acceso potencial y también la continuidad e integralidad de la atención, las barreras económicas identificadas disminuyen el acceso a la consulta médica y por especialistas, a los medicamentos y a las pruebas diagnósticas. Aunque los casos del Rs reportan, mucho más que los del RC, razones económicas para no acceder a los servicios mencionados, estas también son relevantes en el contributivo y favorecen la interrupción en ambos regímenes de los niveles de intervención necesarios en el proceso de recuperación de la salud.

En conclusión y coincidiendo con el estudio de García-Subirats (47), quienes demuestran importantes desigualdades sociales en el uso de servicios en Colombia, relacionadas con el modelo de sistema de salud y revelan, entre otras, diferencias en el uso entre asegurados y no asegurados en los tres niveles asistenciales y entre los regímenes contributivo y subsidiado, los hallazgos presentados en este 176 artículo muestran que estar afiliado al sGsss no implica la misma posibilidad de atención en individuos con necesidades equivalentes dadas por igual estrato social, pues se encontraron diferencias en el acceso a servicios de salud curativos entre la población afiliada al RC y la población afiliada al Rs. Diferencias que se espera puedan ser subsanadas con los cambios que deberán darse en cumplimiento de la Ley Estatutaria del 2015 (12).

En este nuevo contexto, se requiere profundizar en las relaciones necesidad, demanda, oferta y atención de la salud, a nivel nacional, regional y local, con estudios que permitan valorar el impacto o las implicaciones que las nuevas políticas de acceso traerán para mejorar la situación actual.

\section{Referencias bibliográficas}

1. Organización Panamericana de la Salud (ops), Organización Mundial de la Salud (oMs). La rectoría de los ministerios de salud en los procesos de reforma sectorial [documento CD 40/13]. Sesión del 23 de julio de 1977, Tema 5.2 del Programa Provisional. Washington, D.C.: ops/oms, XL Reunión del Consejo Directivo de la Organización Panamericana de la Salud, XLIX Reunión del Comité Regional de la Organización Mundial de la Salud; 1997.

2. Yepes FJ, Ramírez M, Sánchez LH, Ramírez ML, Jaramillo I. Luces y sombras de la reforma de la salud en Colombia. Ley 100 de 1993. Bogotá: Centro Internacional de Investigaciones para el Desarrollo (CIID); 2010.

3. Colombia, Defensoría del Pueblo. La tutela y los derechos a la salud y a la seguridad social 2013. Bogotá, D.C.: Defensoría del Pueblo; 2014.

4. Almeida CM. Reformas de sistemas de servicios de salud y equidad en América Latina y el Caribe: Algunas lecciones de los años 80 y 90. Cad Saude Pública. 2002 Jul.-Ago.; 18 (4): 905-25.

5. Molina-Marín G, Vargas-Jaramillo J, MuñozEcheverry I, Acosta-Gómez J, Sarasti-Vanegas D, Higuita-Higuita Y, et al. Dilemas en las decisiones en la atención en salud. Ética, derechos y deberes constitucionales frente a la rentabilidad financiera en el sistema de salud colombiano. Revista Gerencia y Políticas de Salud. 2010; (18): 103-17.

6. Calderón C, Botero J, Bolaños J, Martínez R. Sistema de salud en Colombia: 20 años de logros y problemas. Cien Saude Colet. 2011; 16 (6): 2817-28.

7. Rodríguez-Acosta S, Trillas F. Barreras y determinantes del acceso a los servicios de salud en Colombia. Barcelona: Universitat Autónoma de Barcelona; 2010. 
8. Congreso de Colombia. Ley 1122 de 2007. Por la cual se hacen ajustes al Sistema General de Seguridad Social en Salud, y se dictan otras disposiciones.

9. Gañán-Echavarría JL. Los muertos de Ley 100. Prevalencia de la libertad económica sobre el derecho fundamental a la salud: una razón de su ineficacia. Caso del Plan Obligatorio de Salud del Régimen Contributivo Medellín: Universidad de Antioquia, Facultad de Derecho y Ciencias Políticas; 2013.

10. Corte Constitucional de Colombia. Sentencia T-760 del 2008. Manuel José Cepeda Jaime Córdoba Triviño, Rodrigo Escobar Gil. Segunda Sala de Revisión.

11. Alcaldía Municipal de Santiago de Cali. Cali en cifras 2013. Santiago de Cali: Departamento Administrativo de Planeación; 2013.

12. Congreso de Colombia. Ley 1751 de 2015. Por medio de la cual se regula el derecho fundamental a la salud y se dictan otras disposiciones.

13. Díaz-Grajales C, Zapata-Bermúdez Y, AristizábalGrisales JC. Acceso a los servicios preventivos en los regímenes contributivo y subsidiado de salud en un barrio estrato dos de la ciudad de Cali. 2011. Rev Gerenc Polit Salud. 2011; 10 (21): 153-75.

14. Garcia-Subirats I, Vargas I, Mogollón-Perez AS, De Paepe P, Ferreira da Silva MR, Unger JP, et al. Inequities in access to health care in different health systems: a study in municipalities of central Colombia and north-eastern Brazil. International Journal for Equity in Health. 2014; 13 (10).

15. Garcia-Subirats I, Vargas I, Mogollón-Pérez AS, De Paepe P, da Silva MRF, Unger JP, Vázquez ML. Barriers in access to healthcare in countries with different health systems. A cross-sectional study in municipalities of central Colombia and north-eastern Brazil. Social Science \& Medicine. 2014; (106): 204-13.

16. Restrepo-Zea JH, Silva-Maya C, Andrade-Rivas F, VH-Dover R. Acceso a servicios de salud: análisis de barreras y estrategias en el caso de Medellín, Colombia. Gerencia y Políticas de Salud. 2014; 13 (27): 242-65.

17. Vargas-Lorenzo I, Vázquez-Navarrete ML, Mogollón-Pérez AS. Acceso a la atención en salud en Colombia. Revista de Salud Pública. 2010; 12 (5): 701-12.

18. Ruiz-Gómez F, Zapata-Jaramillo T, GaravitoBeltrán L. Colombian health care system: results on equity for five health dimensions, 2003-2008. Revista Panamericana de Salud Pública. 2013; 32 (2): 107-15.

19. López-Arana S, Valencia SC, Mogollón AS, Barragán AM, Morales N, Pinilla M, et al. Impacto del modelo de aseguramiento sobre la equidad de acceso a los servicios de salud en Colombia. Salud UIS. 2011; 43 (1): 80-81.

20. Tovar-Cuevas LM, Arrivillaga-Quintero M. Estado del arte de la investigación en acceso a los servicios de salud en Colombia, 2000-2013: revisión sistemática crítica. Rev Gerenc Polít Salud. 2014; 13 (27): 12-26.

21. Mogollón-Pérez AS, Vázquez ML. Factores que inciden en el acceso de la población desplazada a las instituciones prestadoras de servicios de salud en Colombia. Cadernos de Saúde Pública. 2008; 24 (4): 745-54.

22. Mejía-Ortega LM, Hernández-Pacheco J, NietoLópez E. Condiciones de accesibilidad a los servicios de salud de las internas e hijos convivientes en el centro de reclusión para mujeres El Buen Pastor de Medellín, 2009. Revista Gerencia y Políticas de Salud. 2011; 10 (20): 121-37.

23. Trejos-Herrera AM, Tuesca-Molina RdJ, Mosquera-Vásquez M. Niñez afectada con VIH/SIDA: Uso y acceso a servicios de salud en cinco ciudades colombianas. Salud Uninorte. 2011; 25 (2): 5-14.

24. Agudelo-Suárez A, Alzate-Urrea S, López-Vergel F, López-Orozco C, Espinosa-Herrera É, PosadaLópez A, et al. Barreras y facilitadores de acceso a los servicios de salud bucal para la población adulta mayor atendida en la red pública hospitalaria de Medellín, Colombia. Gerencia y Políticas de Salud. 2014; 13 (27): 181-9.

25. Rodríguez-Páez F, Jiménez-Barbosa W, JiménezGonzález C, Coral-Córdoba E, Ramírez-Solano P, Ramos-Navas N. Efecto de las barreras de acceso sobre la asistencia a citas de programa de control prenatal y desenlaces perinatales. Gerencia y Políticas de Salud. 2014; 13 (27): 212-27.

26. Garcés-Palacio I, Rubio-León D, Ramos-Jaraba S. Barreras y facilitadores del sistema de salud relacionadas con el seguimiento de anormalidades citológicas, Medellín-Colombia. Rev Gerenc Polít. Salud. 2014; 13 (27): 200-11.

27. Romero-Prada M, Marrugo-Figueroa R, Acero G, Arango-Bautista C, Alvis-Guzmán N. Impacto en el acceso a medicamentos en la población colombiana después de la actualización del plan de beneficios en el 2012. Gerencia y Políticas de Salud. 2014; 13 (27).

28. Starfield B. Atención primaria. Equilibrio entre necesidad de salud, servicios y tecnología Barcelona: Masson; 1991.

29. Donadebian A. Aspects of medical care administration. Specifying requirements of health care Cambridge, MA: Harvard University Press; 1973.

30. Aday LA, Andersen R. Marco teórico para el estudio del acceso a la atención médica. En White $\mathrm{KL}$, et al. Investigaciones sobre servicios de salud: una antología. Washington, D.C: ops; 1992.

31. Andersen R. Health Status Indices and Access to Medical Care. Am J Public Health. 1978; 68 (5): 458-63.

32. Andersen R. Revisiting the Behavioral Model and Access to Medical Care: Does It Matter. J Health Soc Behav. 1995; 36 (1): 1-10.

33. Penchansky R, Thomas W. The concept of access: Definition and relationship to consumer satisfaction. Med Care. 1981; 19 (2): 127-40.

34. Frenk J. El concepto y la definición de accesibilidad. En White KL, et al. Investigaciones sobre servicios de salud: una antología. Washington, D.C: OPS; 1992.

35. Levesque J, Harris M, Russell G. Patient-Centred Access to Health Care: Conceptualizing Access at the Interface of Health Systems and Populations. 
International Journal for Equity in Health. 2013; 12 (1).

36. Ruiz F, Acosta N, Ardila Z, Eslava J, Peñaloza E, Puente C, et al. Entorno, aseguramiento y acceso en el régimen subsidiado en Colombia. Seis estudios de caso. Bogotá: Fundación Corona, Cendex - Pontificia Universidad Javeriana; 1999.

37. Donadebian A. The quality of care. How can it be assessed? Arch Pathol Lab Med. 1997; (121): 1145-50.

38. Arrendondo A, Meléndez V. Modelos explicativos sobre la utilización de servicios de salud: revisión y análisis. Salud Pública Méx. 1992; 34 (1): 36-48.

39. Silva-Ayçaguer LC. Diseño razonado de muestras y captación de datos para la investigación sanitaria. Madrid: Ediciones Díaz de Santos; 2000.

40. Sugden RA, Smith TMF. Domains of study and poststratification. Journal of Statistical Planning and Inference. 2006; (136): 3307-17.

41. Instituto Nacional de Estadística (INE). Ajuste de los factores de expansión por postestratificación. Programa para el mejoramiento de las encuestas y la medición de las condiciones de vida en América Latina y El Caribe, Mecovi [internet] [acceso: 1으 de noviembre del 2007]. Disponible en: http://www. eclac.cl/deype/mecovi/taller14.htm.

42. Mejía-Mejía A, Sánchez-Gandur AF, TamayoRamírez JC. Equidad en el acceso a servicios de salud en Antioquia, Colombia. Rev Salud Pública. 2007; 9 (1): 26-38.
43. Varela A, Carrasquilla G, Tono T, Samper B. Asimetría en la información: barreras para la implementación de la reforma de salud en Colombia. Colombia Médica. 2002; 33 (3).

44. Rubio-Mendoza ML. Equidad en el acceso a los servicios de salud y equidad en la financiación de la atención en Bogotá. Rev Salud Pública. 2008; (10): 29-43.

45. O’Meara-Bautista G, Ruiz-Gómez F, Amaya-Lara J. Impacto del aseguramiento sobre uso y gasto en salud en Colombia. Bogotá: Pontificia Universidad Javeriana; 2003.

46. Alvis-Estrada L, Alvis-Guzmán N, De la Hoz F. Gasto privado en salud de los hogares de Cartagena de Indias, 2004. Rev. Salud Pública [serie en internet] 2007 [acceso: 13 de junio del 2010]; 9 (1): 11-25. Disponible en: http://www.scielo.org. co/scielo.php? script $=$ sci_arttext $\&$ pid $=$ S0124 00642007000100004\&lng = es.

47. Garcia-Subirats I, Vargas-Lorenzo I, MogollónPérez AS, Paepe PD, Ferreira-da Silva MR, Unger JP, et al. Determinantes del uso de distintos niveles asistenciales en el Sistema General de Seguridad Social en Salud y Sistema Único de Salud en Colombia y Brasil. Gac Sanit [serie en internet]. 2014 Dic; [acceso: 24 de agosto del 2015]. 28 (6): 480-8. Disponible en: http://scielo. isciii.es/scielo.php?script $=$ sci_arttext\&pid $=\mathrm{S} 0213$ 91112014000600008\&lng=es. ttp://dx.doi. org/10.1016/j.gaceta.2014.05.010. 